\title{
Citrate Anticoagulation for Continuous Renal Replacement Therapy in Critically Ill Patients: Success and Limits
}

\author{
Filippo Mariano, ${ }^{1}$ Daniela Bergamo, ${ }^{1}$ Ezio Nicola Gangemi, ${ }^{2}$ Zsuzsanna Hollo', 1 \\ Maurizio Stella, ${ }^{2}$ and Giorgio Triolo ${ }^{1}$ \\ ${ }^{1}$ Department of Medicine Area, Nephrology and Dialysis Unit, CTO Hospital, Via G. Zuretti 29, Turin, Italy \\ ${ }^{2}$ Department of Plastic Surgery, Burns Unit, CTO Hospital, 10126 Turin, Italy \\ Correspondence should be addressed to Filippo Mariano, filippo.mariano@poste.it
}

Received 30 September 2010; Accepted 20 January 2011

Academic Editor: Michael Oppert

Copyright (C) 2011 Filippo Mariano et al. This is an open access article distributed under the Creative Commons Attribution License, which permits unrestricted use, distribution, and reproduction in any medium, provided the original work is properly cited.

\begin{abstract}
Citrate anticoagulation has risen in interest so it is now a real alternative to heparin in the ICUs practice. Citrate provides a regional anticoagulation virtually restricted to extracorporeal circuit, where it acts by chelating ionized calcium. This issue is particularly true in patients ongoing CRRT, when the "continuous" systemic anticoagulation treatment is per se a relevant risk of bleeding. When compared with heparin most of studies with citrate reported a longer circuit survival, a lower rate of bleeding complications, and transfused packed red cell requirements. As anticoagulant for CRRT, the infusion of citrate is prolonged and it could potentially have some adverse effects. When citrate is metabolized to bicarbonate, metabolic alkalosis may occur, or for impaired metabolism citrate accumulation leads to acidosis. However, large studies with dedicated machines have indeed demonstrated that citrate anticoagulation is well tolerated, safe, and an easy to handle even in septic shock critically ill patients.
\end{abstract}

\section{Introduction}

Multiple technological advancements affecting continuous renal replacement therapy (CRRT) delivery to critically ill patients have been developed in the past twenty years. Dedicated CRRT equipment with better thermal control, more precise balances and volumetric control of infused and filtered fluids, and user friendly machines now allow a safe CRRT provision, which has become increasingly popular.

Generally speaking, the practical background of continuous treatment feasibility in critically ill patients, often hemodynamically instable, septic, or suffering from trauma or recent surgery, is closed to the need for anticoagulation. In effect, systemic anticoagulation is still the main challenge in the application of CRRT, since it exposes the patient to a risk of active bleeding episodes.

Heparin, first adopted as an anticoagulant in the late 1920s, made feasible the patency of extracorporeal circuits, and nowadays it remains the most popular anticoagulant worldwide used in extracorporeal dialysis [1]. Heparin is the drug of choice in chronic patients undergoing hemodialysis as well as in acute patients treated by CRRT. Heparin is efficient and instantaneous in its anticoagulation, quite safe and cheap so that heparin can be administered with ease to patients.

Bleeding is the main side effect of i.v. heparin administration for CRRT. The incidence of bleeding episodes considering all of the administration methods ranges from $10 \%$ to $50 \%$, with a bleeding mortality rate as high as $15 \%$ [2-4]. Heparin is contraindicated in critically ill patients with active bleeding or at high risk of bleeding, as seen in such patients with extensive trauma, burns, or in surgery patients [2].

As alternative to heparin, several methods of systemic or regional anticoagulation have been proposed over the past 50 years, including low-molecular weight heparin, prostacyclin, the serine proteinase inhibitor nafamostat, hirudin, regional heparinization, saline flushes, and regional citrate anticoagulation. Among these, citrate anticoagulation has risen in interest so it is now a real alternative to heparin in the ICUs practice of CRRT. 


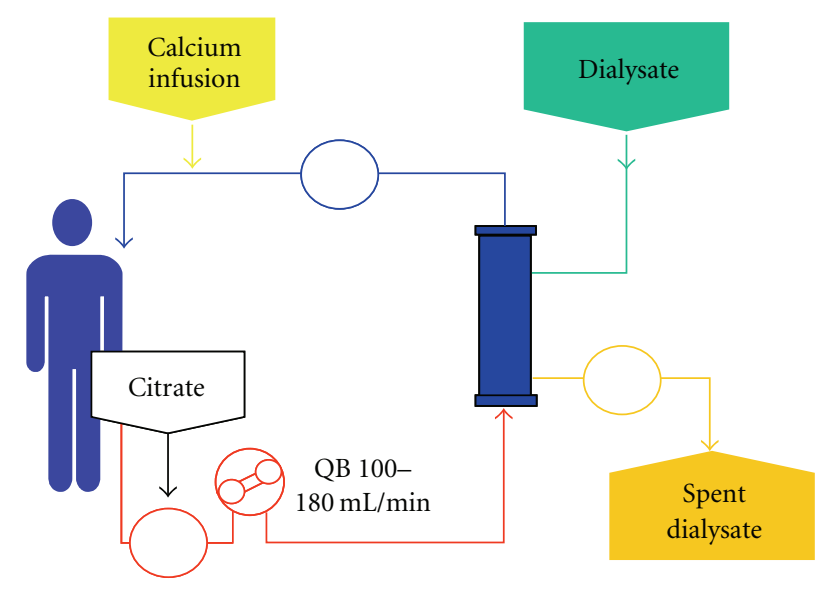

FIGURE 1: Scheme of standard citrate anticoagulation in CVVHDF.

\section{Regional Citrate Anticoagulation as Alternative to Heparin}

Citrate was first reported as an anticoagulant for hemodialysis in 1960s by Morita et al. [5] and as an alternative regional anticoagulation in patients ongoing CRRT in 1990 by Metha et al. [6]. Since then, citrate has gained more and more popularity. Regional citrate anticoagulation has been utilized in CRRT programmes based on its fundamental properties of avoiding a systemic anticoagulation. Citrate provides a regional anticoagulation virtually restricted to extracorporeal circuit, where it acts by chelating ionized calcium. This issue is particularly true in patients ongoing CRRT, when the "continuous" systemic anticoagulation treatment is per se a relevant risk of bleeding.

However, citrate use is not uniform, and it has been utilized mainly in North America and Europe CRRT programmes. For instance, anticoagulation with a regional citrate or systemic heparin standardized protocol for CRRT has been implemented since 1999 in all adult ICUs patients in Canadian Calgary Health Region [7]. Based on a North American survey, it has been estimated that a quarter of all patients suffering from acute kidney injury (AKI) are treated with CRRT, and regional citrate anticoagulation has been used as method of choice in only $13 \%$ of these patients [8]. In a recent survey on all ICUs practice in North-West of Italy (covering a population of 4.5 millions of inhabitants), in the vast majority of dialysis sessions done in 2007 unfractionated heparin was the anticoagulant of choice $(5,296$ out of 7,842 dialysis sessions, $67.5 \%$ ). Interestingly, on patients at high risk of bleeding regional citrate anticoagulation was performed only in $18.0 \%$ of the cases, whereas the principle treatment modality remained that of a dialysis session without heparin, or at low heparin doses with saline flushes $(77.6 \%$ ) [9]. However, it is reasonable to presume that the use of citrate will grow in popularity in the near future. The inherent complexities of the method are now reduced since RRT dedicated monitor may indeed provide a safe and an easy-to-handle citrate anticoagulation protocol besides standard heparin anticoagulation [10].

\section{Citrate Acts by Reducing Ionized Calcium $\left(\mathrm{iCa}^{++}\right)$Concentration}

As anticoagulant, citrate has been applied to hemodialysis, hemodiafiltration (in pre/postdilution), hemofiltration (in pre-postdilution), sorbent technology and in both continuous and intermittent treatments $[4,6,7,11-20]$. By a Medline search more than 60 different systems for citrate administration can be found. Most of these systems have been home made with its specific composition of fluids, and its own rules to provide anticoagulation and titrate calcium administration. In the last years global citrate market has been deeply changing. Dialysis machines for CRRT have incorporated citrate anticoagulation in the soft- and hardware, as well as dedicated fluids have been certified and registered by industries.

However, as shown in Figure 1 all citrate systems present in the market work on few simple and shared modalities:

(1) prefilter infusion of citrate, which acts as chelating ionized calcium (an iCa concentration below $0.35 \mathrm{mmol} / \mathrm{L}$ is required to inhibit coagulation);

(2) dialysate (and predilution or postdilution if present) fluids are calcium free;

(3) a replacement infusion of calcium at the end of extracorporeal circuit, in the blood line returning to patient.

As part of mechanisms operating during citrate anticoagulation, in the filter citrate-calcium complex and $\mathrm{iCa}^{++}$ are partially cleared by convection and/or diffusion, since membranes currently used in CRRT have a sieving coefficients near to unit for these small molecules. The remaining amount of citrate not cleared by filter enters the systemic circulation, and should be metabolized. As a consequence of the use of calcium free fluids, citrate anticoagulation leads to a net loss of calcium by filter. In the patient $\mathrm{iCa}^{++}$rises again by replacement infusion of calcium at the end of circuit and the liberation of chelated calcium when citrate is metabolised.

\section{Efficacy and Safety of Citrate}

4.1. Circuit Survival. In respect to heparin, citrate is emerging as being similar or even superior as anticoagulant in terms of filter life and efficacy of anticoagulation. Factors influencing the circuit life reflect in part the coagulation capacity of the patient (procoagulant and/or anticoagulant factors due to acute illness, platelet count) and in part the technical aspects of dialysis (diffusion/convection modality, filtration fraction, pre- postdilution, vascular access quality, blood flow rate, nursing monitoring of dialysis).

In addition, citrate anticoagulation could be modulated by the target of citrate concentration reached in circuit blood (from 3 to $5 \mathrm{mmol} / \mathrm{L}$ of citratemia). As increased levels of anticoagulation could be obtained according to increased dose of heparin, a level of citratemia of $5 \mathrm{mmol} / \mathrm{L}$ (according to a concentration of $\mathrm{iCa}^{++}$below $0.1 \mathrm{mmol} / \mathrm{L}$ ) leads to a total and persistent inability of blood coagulation. 
Most of studies reported longer circuit survival with citrate, but only few studies comparing citrate to heparin were randomized [13,14, 21-23]. Circuit life was significantly longer with citrate $[13,14,23]$, while circuit life was similar in the other $2[21,22]$. As a matter of fact, blood citrate concentration of around $4 \mathrm{mmol} / \mathrm{L}$ is adapted to achieve excellent filter run times. Some trials who found that citrate did not achieve better filter run time used a blood citrate concentration of $3 \mathrm{mmol} / \mathrm{L}$, and often did not control the ionized postfilter calcium values [21].

In a prospective, randomized clinical trial, Monchi et al. compared unfractionated heparin and regional citrate anticoagulation in $20 \mathrm{CVVH}$ patients [13]. They found a median circuit life of $40 \mathrm{~h}$ with heparin and $70 \mathrm{~h}$ with citrate (heparin adjusted to get an APTT at 60-80 s, citrate infusion adjusted to maintain circuit $\mathrm{iCa}^{++}<0.3 \mathrm{mmol} / \mathrm{L}$ ). Similarly, a prospective randomized study involving 30 patients undergoing CVVHDF not at high risk of hemorrhagic complications showed a median hemofilter survival time of 124.5 hours in the citrate group and 38.3 hours in the heparin group $(P<.001)$ [22]. Heparin dose was adjusted to obtain an APTT at $45-65 \mathrm{~s}$, citrate infusion to maintain circuit $\mathrm{iCa}^{++}$between $0.25-0.35 \mathrm{mmol} / \mathrm{L}$. In a recent prospective randomized multicenter trial Hetzel et al. [23] compared in CVVHF in total predilution citrate with heparin. Mean hemofilter patency was significantly longer in the HF-Citrate group compared with the HF-bicarbonate group $(37.5 \pm 23 \mathrm{~h}$ versus $26.1 \pm 19 \mathrm{~h}, P<.001, n=$ $87 / 81)$.

In contrast, in other 2 randomized trials citrate was not superior to heparin in circuit survival [21, 22]. In a large multi-center study involving seven USA centers, 138 patients and 442 CRRT circuits were studied to assess filter life span and anticoagulation complications with anticoagulation based on heparin, citrate, or with no anticoagulation [15]. Mean circuit survival was not different for circuits receiving heparin $(42.1 \pm 27.1 \mathrm{~h})$ and citrate $(44.7 \pm 35.9 \mathrm{~h})$, with similar clotting rates and without any significant difference by Kaplan-Meier analyses of survival between the two groups. Circuits without anticoagulation presented a significant lower survival $(27.2 \pm 21.5 \mathrm{~h}, P<.001)$.

In another study of 87 patients undergoing CRRT from the Calgary Health Region, Canada, 54 were initially treated with citrate (212 filters), 29 with heparin (97 filters), and 4 with saline flushes [7]. Median filter lifespan was significantly higher with citrate than with UFH (40 hours versus, 30 hours, $P<.001$ ) [7].

Finally, in 70 severe burn patients with septic shock treated by continuous or intermittent HDF with citrate or heparin anticoagulation, circuit survival was significantly longer with citrate on continuous treatment but not in intermittent modality [24].

4.2. Bleeding Complications. The primary reason to use citrate is that it leads to a regional anticoagulation, virtually restricted to extracorporeal circuit. Therefore, citrate anticoagulation does not increase patient risk of bleeding. In addition citrate is specifically indicated in patient at high risk of bleeding.
The 5 available randomized studies comparing heparin to citrate enrolled patients excluding those at high bleeding risk [13, 14, 21-23].

However, bleeding episodes were similar with citrate and heparin in two reports $[13,21]$ and reduced with citrate in other two $[14,22,23]$. No definite hemorrhage in the citrate group and seven instances in the heparin group and one occult hemorrhage in both citrate and heparin groups were observed [14]. After adjustment for antithrombin-III levels and illness severity score, the relative risk of hemorrhage with citrate anticoagulation was still significantly lower than that with heparin $(0.14$ versus 0.96$)$. Even if citrate was used in presence of a lower systemic anticoagulation with heparin (at mean dose of 5,428 UI/day), bleeding complications episodes $(5.7 \%)$ were significantly lower in comparison with heparin ( $14.5 \%$ episodes, at mean heparin dose of $13,174 \mathrm{UI} /$ day) [23].

Similarly, the number of transfused packed red cells per day with citrate was similar to those with heparin in 3 studies $[14,21,22]$, whereas in CVVHF study by Monchi et al. [13] was significantly reduced (1.0 transfused packed red cells/day in heparin group and 0.2 in citrate group).

In the multi-center study involving 138 patients and 442 CRRT circuits, life-threatening bleeding complications as a result of the anticoagulant were shown in 9 patients in the heparin group and absent in citrate group [15]. In the same way, in a study of 87 patients undergoing CRRT from Calgary Health Region citrate anticoagulation was well tolerated, and no treatment was discontinued for hemorrhagic episodes [7].

In 70 severe burn patients with septic shock and AKI, and undergoing RRT, bleeding complications were significantly lower with citrate during CVVHDF, as well as the requirements of transfused packed red cells per day ( 1.76 transfused packed red cells/day with heparin group and 0.98 with citrate group) [24].

\subsection{Citrate Anticoagulation in Critically Ill Patients Treated} with Sorbent Technology. Sorbent technology is a promising tool for extracorporeal techniques targeted to remove detrimental substances present in blood of septic shock patients. Sorbents have specific characteristics, such as an access to substances bound to protein, a removal capacity of larger molecular weight toxins exceeding cut-off of dialysis membranes. In addition, in the future many advances in resin sorbent technology will come out.

As anticoagulant, citrate has been demonstrated to be safe and efficient in septic shock patients treated by sorbent technology [25]. In 13 severe burn and polytrauma patients at high risk or with active bleeding and treated with plasma adsorption, 58 sessions using systemic anticoagulation with heparin (mean heparin $741 \mathrm{U} / \mathrm{h}$ ) were compared with 28 sessions using citrate regional anticoagulation (circuit citratemia at $4 \mathrm{mmol} / \mathrm{L}$ ) evaluating efficiency and safety of the technique. Plasma filtration efficiency and number of used cartridges were similar, whereas the number of lost cartridges was significantly lower in the citrate patients [25]. 


\section{Metabolic Consequences and Tolerance of Citrate}

When administered intravenously in healthy subjects citrate is rapidly metabolized to bicarbonate by the tricarboxylic pathway in liver, kidney, and skeletal muscle. In addition, at high serum levels a substantial percentage of citrate is excreted unchanged in the urine [26]. Citrate can also be involved in many other biochemical pathways including amino acid synthesis and gluconeogenesis [26].

In presence of chronic renal failure citrate metabolism may be impaired. Both loss of metabolically active kidney mass and accumulation of soluble toxins can affect some metabolic pathways in extrarenal tissues. In addition, liver metabolism of gluconeogenetic intermediates and the tricarbonic acid cycle are impaired in uremic state [27]. Investigating the metabolism of citrate in patients on regular hemodialysis and minimal residual renal function (urinary output $<400 \mathrm{~mL} / \mathrm{d}$ ), Bauer et al. [27] did not observe alkalinization or $\mathrm{pH}$ variation after sodium citrate i.v. infusion.

When used for regional anticoagulation of the dialysis circuit in CRRT, the infusion of citrate is prolonged, and it may have some adverse effects. Citrate is an organic compound able to react with divalent cations such as magnesium and calcium. By chelating calcium citrate blocks coagulation activation in the dialysis circuit. As citrate is metabolized to bicarbonate, metabolic alkalosis may occur or, in contrast, if citrate is not metabolized and accumulates, acidosis can develop. Moreover, since citrate is usually applied as trisodium citrate it can lead to excessive sodium load and hypernatremia. Hypomagnesemia may be another consequence but, at our knowledge, no case of severe hypomagnesemia has been reported.

In 1997 Palsson and Niles [11] reported 2 cases of citrate accumulation and refractory systemic hypocalcemia using a simplified protocol with prefilter citrate infusion during CVVHF in 15 patients. More recently by using the same protocol as citrate as the only buffer substance Hetzel et al. [23] found equivalence of standard bicarbonate in groups HF-citrate and HF-bicarbonate from day 3 to day 11. However, more patients in the HF-citrate group needed additional bicarbonate infusions compared with the patients treated with heparin.

In a large single-center analysis of 209 patients (37 received citrate as sole anticoagulant, 87 low-dose heparin plus citrate, and 85 only heparin), a development of metabolic alkalosis in $50 \%$ of patients treated with citrate was observed [18], and all cases were solved by increasing the dialysate flow rate.

Three recently controlled large studies [7, 14, 15], involving a total of 251 patients and comparing citrate (121 patients) with heparin anticoagulation in CRRT in critically ill patients, confirmed the safety of the citrate. In 138 patients and 442 CRRT circuits, out of 37 patients treated with citrate, metabolic alkalosis was shown in 4 cases and citrate accumulation in 2 patients with liver failure but they were all managed by decreasing infusion rate of bicarbonate containing solution or of citrate [15]. Generally speaking, citrate anticoagulation was well tolerated, and no treatment was discontinued for hypernatremia, metabolic alkalosis, hypocalcemia, or citrate accumulation. A safe and an easyto-handle citrate anticoagulation protocol now commercially available has been recently validated in 162 patients [20]. This protocol provided an excellent acid base and electrolyte control in critically ill patients with acute renal failure, allowing a wide spectrum of treatment doses.

Liver is the most important organ in citrate metabolism. Kramer et al. [28] showed that in intensive care patients liver citrate clearance is decreased by approximately $50 \%$ in patients with cirrhosis compared with normal liver function. In cirrhotic patients impairment of citrate metabolism can cause citrate increase and hypercalcemia due to an accumulation of calcium-citrate complexes [28].

When citrate-calcium complexes increase in circulation, systemic $\mathrm{iCa}^{++}$concentration decreases and the ratio between total to ionized calcium increases [29]. Ratio has been used as an indirect parameter of blood citrate increase. However, this ratio may not predict citrate accumulation in all cases $[28,30]$.

A recent study [24] involving 31 severe burn patients undergoing CVVHDF or SLED-HDF with citrate regional anticoagulation showed a good metabolic tolerance. Systemic arterial $\mathrm{pH}, \mathrm{Na}^{+}, \mathrm{K}^{+}, \mathrm{iCa}^{++}$, total $\mathrm{Ca}^{++} / \mathrm{iCa}^{++}$ratio and bicarbonates did not show any derangements over the period of observation. Citrate was determined directly dosing systemic citratemia and the levels of citrate in the ultrafiltrate. During the CRRT a high portion of the citratecalcium complexes were lost in effluent (membranes currently used were highly permeable to free and calcium-bound citrate, with a sieving coefficients near to unit). The marked loss of citrate (up to $60 \%$ of the infused amount) directly correlated with effluent volume. By increasing dialysate flow rate, citrate loss could be increased [24] and decreased the remaining amount of citrate-calcium complexes returning to the patient.

Oudemans-van Straaten [21] recently compared the safety and efficacy of citrate with the nadroparin anticoagulation in 200 patients (97 treated with citrate) on CRRT. As it concerns tolerance citrate was superior to low-molecular weight heparin. Nadroparin patients more frequently developed metabolic alkalosis, hyponatremia and hyperlactatemia, whereas initial hypocalcemia was less often corrected in the citrate patients. Unexpectedly, citrate seemed to improve patient and kidney survival (three-month mortality was $48 \%$ with citrate versus $63 \%$ with nadroparin). Citrate appeared particularly beneficial in the subgroups of patients after surgery, with sepsis or with severe multiple organ failure [21]. However, favourable data about mortality are not confirmed in more recent multicenter randomized trial [23]. Sepsis was the predominant reason for death in both studies [21, 23], and exposure to citrate was considerably longer ( 8.5 days versus 2.7 days) in the trial without any favourable effect on mortality [23].

\section{References}

[1] G. Haas, "Uber Blutwaschung," Klinische Wochenschrift, vol. 7, no. 29, pp. 1356-1362, 1928. 
[2] J. Van de Watering, R. G. J. Westendorp, J. G. van der Hoeven, B. Stolk, J. D. M. Feuth, and P. C. Chang, "Heparin use in continuous renal replacement procedures: the struggle between filter coagulation and patient hemorrhage," Journal of the American Society of Nephrology, vol. 7, no. 1, pp. 145-150, 1996.

[3] P. Y. Martin, J. C. Chevrolet, P. Suter, and H. Favre, "Anticoagulation in patients treated by continuous venovenous hemofiltration: a retrospective study," American Journal of Kidney Diseases, vol. 24, no. 5, pp. 806-812, 1994.

[4] L. Gabutti, C. Marone, G. Colucci, F. Duchini, and C. Schönholzer, "Citrate anticoagulation in continuous venovenous hemodiafiltration: a metabolic challenge," Intensive Care Medicine, vol. 28, no. 10, pp. 1419-1425, 2002.

[5] Y. Morita, R. W. Johnson, R. E. Dorn, and D. S. Hall, "Regional anticoagulation during hemodialysis using citrate," The American Journal of the Medical Sciences, vol. 242, pp. 3243, 1961.

[6] R. L. Mehta, B. R. McDonald, M. M. Aguilar, and D. M. Ward, "Regional citrate anticoagulation for continuous arteriovenous hemodialysis in critically ill patients," Kidney International, vol. 38, no. 5, pp. 976-981, 1990.

[7] S. M. Bagshaw, K. B. Laupland, P. J. E. Boiteau, and T. Godinez-Luna, "Is regional citrate superior to systemic heparin anticoagulation for continuous renal replacement therapy? A prospective observational study in an adult regional critical care system," Journal of Critical Care, vol. 20, no. 2, pp. 155-161, 2005.

[8] A. Hyman and D. C. Mendelssohn, "Current Canadian approaches to dialysis for acute renal failure in the ICU," American Journal of Nephrology, vol. 22, no. 1, pp. 29-34, 2002.

[9] F. Mariano, M. Pozzato, G. Canepari, C. Vitale, F. Bermond, and C. Sacco, "Renal replacement therapy in intensive care units: a survey of nephrological practice in northwest Italy," Journal of Nephrology, vol. 24, no. 2, pp. 165-176, 2011.

[10] C. S. C. Bouman, "And the winner is: regional citrate anticoagulation," Critical Care Medicine, vol. 37, no. 2, pp. 764-765, 2009.

[11] R. Palsson and J. L. Niles, "Regional citrate anticoagulation in continuous venovenous hemofiltration in critically ill patients with a high risk of bleeding," Kidney International, vol. 55, no. 5, pp. 1991-1997, 1999.

[12] A. J. Tolwani, R. C. Campbell, M. B. Schenk, M. Allon, and D. G. Warnock, "Simplified citrate anticoagulation for continuous renal replacement therapy," Kidney International, vol. 60, no. 1, pp. 370-374, 2001.

[13] M. Monchi, D. Berghmans, D. Ledoux, J. L. Canivet, B. Dubois, and P. Damas, "Citrate vs. heparin for anticoagulation in continuous venovenous hemofiltration: a prospective randomized study," Intensive Care Medicine, vol. 30, no. 2, pp. 260-265, 2004.

[14] D. J. Kutsogiannis, R. T. N. Gibney, D. Stollery, and J. Gao, "Regional citrate versus systemic heparin anticoagulation for continuous renal replacement in critically ill patients," Kidney International, vol. 67, no. 6, pp. 2361-2367, 2005.

[15] P. D. Brophy, M. J. G. Somers, M. A. Baum et al., "Multicentre evaluation of anticoagulation in patients receiving continuous renal replacement therapy (CRRT)," Nephrology Dialysis Transplantation, vol. 20, no. 7, pp. 1416-1421, 2005.

[16] S. W. Tobe, P. Aujla, A. A. Walele et al., "A novel regional citrate anticoagulation protocol for CRRT using only commercially available solutions," Journal of Critical Care, vol. 18, no. 2, pp. 121-129, 2003.
[17] O. Cointault, N. Kamar, P. Bories et al., "Regional citrate anticoagulation in continuous venovenous haemodiafiltration using commercial solutions," Nephrology Dialysis Transplantation, vol. 19, no. 1, pp. 171-178, 2004.

[18] S. Morgera, C. Scholle, G. Voss et al., "Metabolic complications during regional citrate anticoagulation in continuous venovenous hemodialysis: single-center experience," Nephron, vol. 97, no. 4, pp. c131-c136, 2004.

[19] A. J. Tolwani, M. B. Prendergast, R. R. Speer, B. S. Stofan, and K. M. Wille, "A practical citrate anticoagulation continuous venovenous hemodiafiltration protocol for metabolic control and high solute clearance," Clinical Journal of the American Society of Nephrology, vol. 1, no. 1, pp. 79-87, 2006.

[20] S. Morgera, M. Schneider, T. Slowinski et al., "A safe citrate anticoagulation protocol with variable treatment efficacy and excellent control of the acid-base status," Critical Care Medicine, vol. 37, no. 6, pp. 2018-2024, 2009.

[21] H. M. Oudemans-van Straaten, R. J. Bosman, M. Koopmans et al., "Citrate anticoagulation for continuous venovenous hemofiltration," Critical Care Medicine, vol. 37, no. 2, pp. 545$552,2009$.

[22] M. G. H. Betjes, D. van Oosterom, M. van Agteren, and J. van de Wetering, "Regional citrate versus heparin anticoagulation during venovenous hemofiltration in patients at low risk for bleeding: similar hemofilter survival but significantly less bleeding," Journal of Nephrology, vol. 20, no. 5, pp. 602-608, 2007.

[23] G. R. Hetzel, M. Schmitz, H. Wissing et al., "Regional citrate versus systemic heparin for anticoagulation in critically ill patients on continuous venovenous haemofiltration: a prospective randomized multicentre trial," Nephrology Dialysis Transplantation, vol. 26, no. 1, pp. 232-239, 2011.

[24] F. Mariano, L. Tedeschi, M. Morselli, M. Stella, and G. Triolo, "Normal citratemia and metabolic tolerance of citrate anticoagulation for hemodiafiltration in severe septic shock burn patients," Intensive Care Medicine, vol. 36, no. 10, pp. 1735-1743, 2010.

[25] F. Mariano, C. Tetta, M. Stella, P. Biolino, A. Miletto, and G. Triolo, "Regional citrate anticoagulation in critically ill patients treated with plasma filtration and adsorption," Blood Purification, vol. 22, no. 3, pp. 313-319, 2004.

[26] G. Nuthall, P. Skippen, C. Daoust, F. Al-Jofan, and M. Seear, "Citrate anticoagulation in a piglet model of pediatric continuous renal replacement therapy," Critical Care Medicine, vol. 30, no. 4, pp. 900-903, 2002.

[27] E. Bauer, K. Derfler, C. Joukhadar, and W. Druml, "Citrate kinetics in patients receiving long-term hemodialysis therapy," American Journal of Kidney Diseases, vol. 46, no. 5, pp. 903907, 2005.

[28] L. Kramer, E. Bauer, C. Joukhadar et al., "Citrate pharmacokinetics and metabolism in cirrhotic and noncirrhotic critically ill patients," Critical Care Medicine, vol. 31, no. 10, pp. 24502455, 2003.

[29] H. U. Meier-Kriesche, J. Gitomer, K. Finkel, and T. DuBose, "Increased total to ionized calcium ratio during continuous venovenous hemodialysis with regional citrate anticoagulation," Critical Care Medicine, vol. 29, no. 4, pp. 748-752, 2001.

[30] G. R. Hetzel, G. Taskaya, C. Sucker, M. Hennersdorf, B. Grabensee, and M. Schmitz, "Citrate plasma levels in patients under regional anticoagulation in continuous venovenous hemofiltration," American Journal of Kidney Diseases, vol. 48, no. 5, pp. 806-811, 2006. 


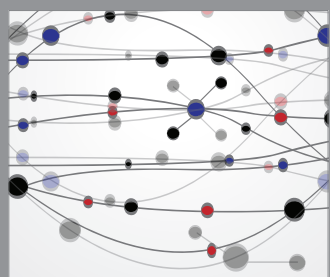

The Scientific World Journal
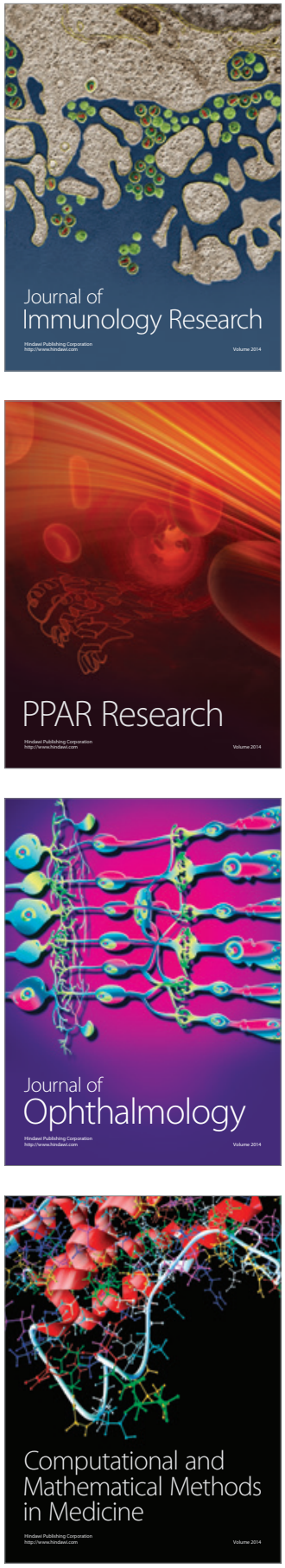

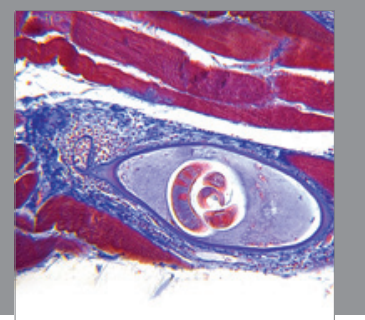

Gastroenterology

Research and Practice
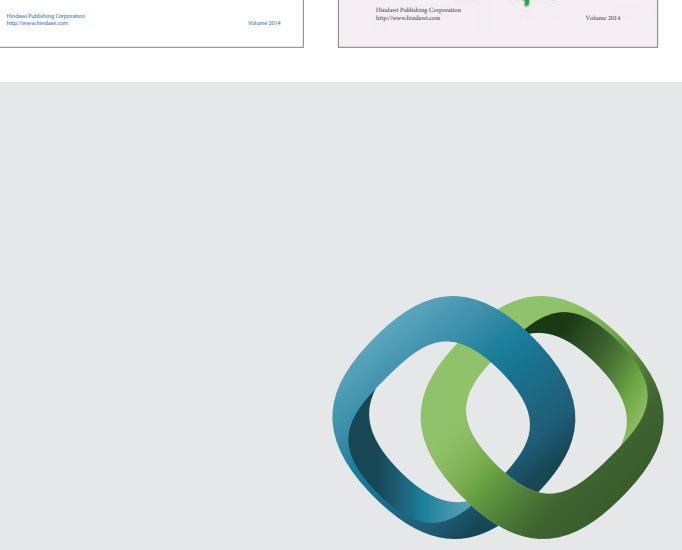

\section{Hindawi}

Submit your manuscripts at

http://www.hindawi.com
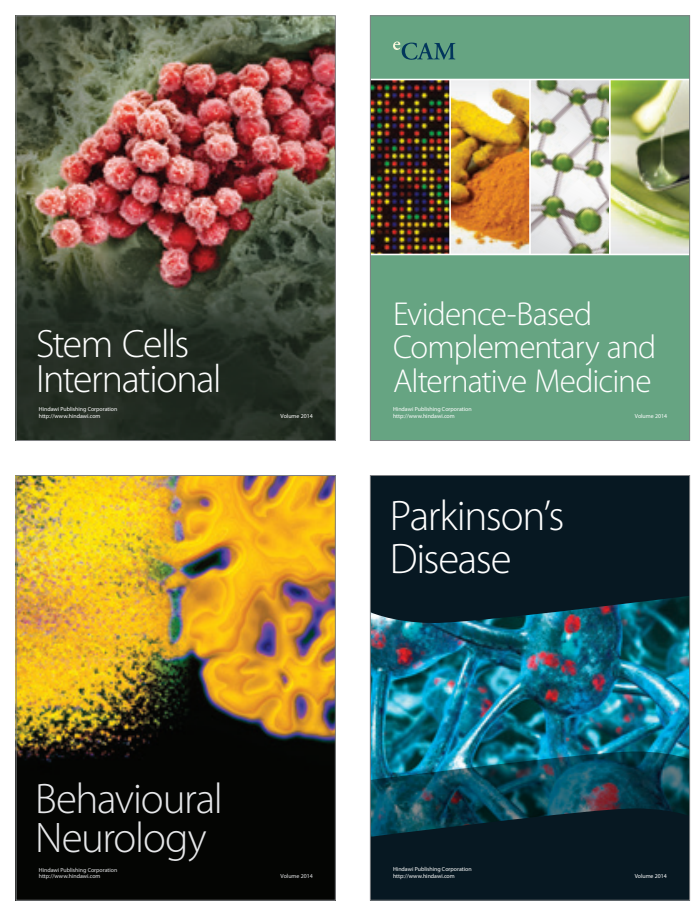

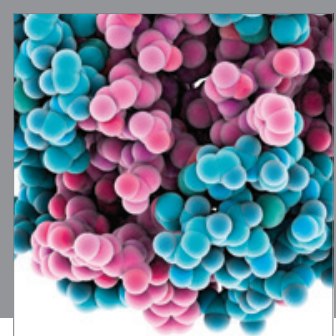

Journal of
Diabetes Research

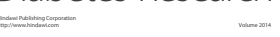

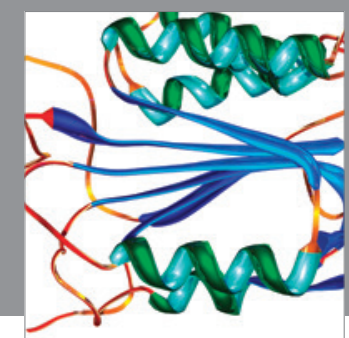

Disease Markers
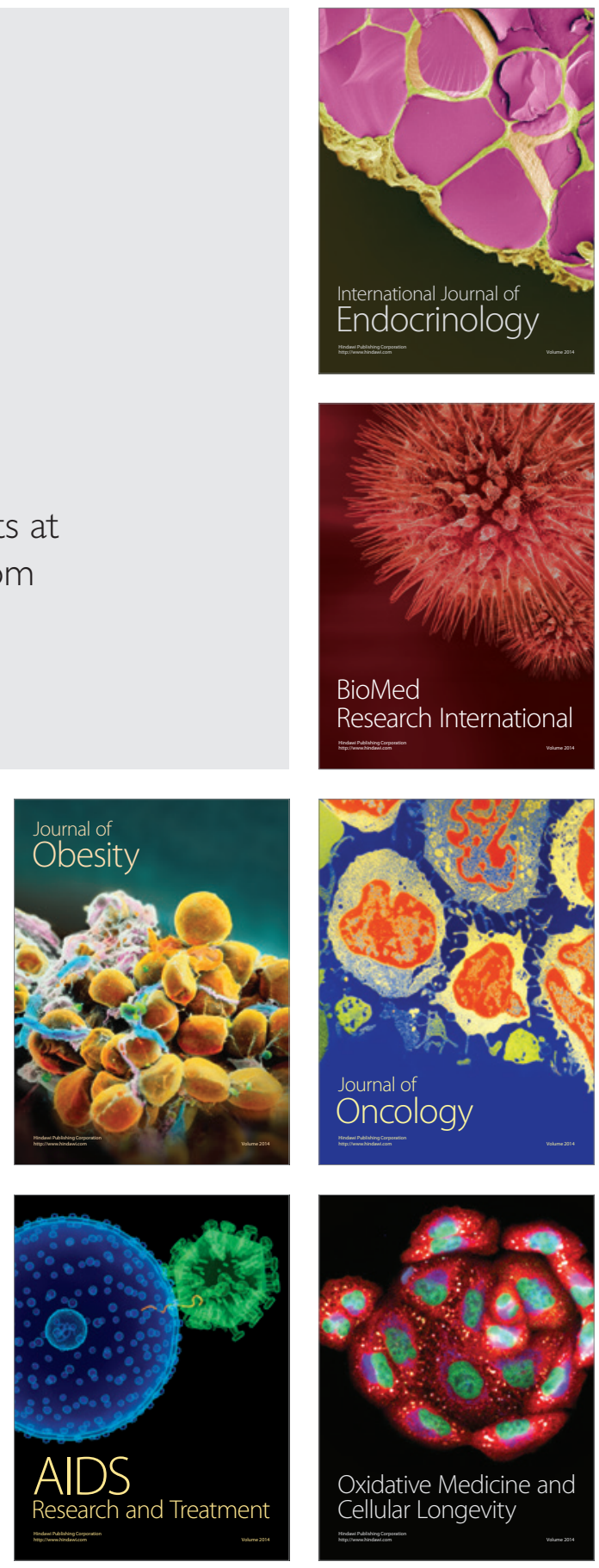